\title{
Relationship Between Self-Reported Concomitant Depressive and Anxiety Symptoms and the Post-Concussion Symptoms Scale (PCSS)
}

\author{
Garrett A. Thomas* (D) Kaitlin E. Riegler (D), Erin T. Guty (D) and Peter A. Arnett (D) \\ Department of Psychology, The Pennsylvania State University, University Park, PA, USA
}

(Received April 19, 2021; Final Revision September 15, 2021; Accepted October 20, 2021; First Published Online December 13, 2021)

\begin{abstract}
Objectives: The current study explored how affective disturbances, particularly concomitant anxiety and depressive symptoms, impact baseline symptom self-reporting on the Post-Concussion Symptoms Scale (PCSS) in college athletes. Methods: Athletes were separated into four groups (Healthy Control $(\mathrm{HC})(n=581)$, Depression Only $(n=136)$, Anxiety Only $(n=54)$, Concomitant Depression/Anxiety $(n=62))$ based on their anxiety and depression scores. Groups were compared on Total PCSS Score as well as 5 PCSS Symptom Cluster scores (Cognitive, Physical, Affective, Sleep, and Headache). Results: The three affective groups reported significantly greater symptomatology than HCs, with the Concomitant group showing the highest symptomatology scores across all clusters. The depressive symptoms only group also reported significantly elevated symptomatology, compared to HCs, on every symptom cluster except headache. The anxiety symptoms only group differed from HCs on only the cognitive symptoms cluster. Additionally, the Concomitant group reported significantly increased PCSS symptomatology, in terms of total scores and all 5 symptom clusters, compared to the depressive symptoms only and anxiety symptoms only groups. Conclusions: Our findings suggest that athletes experiencing concomitant depressive/anxiety symptoms report significantly greater levels of symptomatology across all 5 PCSS symptom clusters compared to HCs. Further, results suggest that athletes experiencing concomitant affective disturbance tend to report greater symptomatology than those with only one affective disturbance. These findings are important because, despite the absence of concussion, the concomitant group demonstrated significantly elevated symptomatology at baseline. Thus, future comparisons with post-concussion data should account for this increased symptomatology, as test results may be skewed by affective disturbances at baseline.
\end{abstract}

Keywords: Depression, anxiety, comorbidity, affective symptoms, post-concussion symptoms, athletics

\section{INTRODUCTION}

Assessing self-reported symptomatology is an important aspect of concussion management as most clinicians will not allow athletes to return to play if they are still symptomatic, even if they are deemed "neurocognitively recovered." For example, Arnett and colleagues (2016) laid out an empirically derived algorithm for determining neurocognitive recovery, yet progression to the next steps following "recovery" is dependent upon an absence/reduction of symptoms as determined by self-report. However, what should one do if athletes report elevated symptoms, even at baseline when they are not concussed? Is it still appropriate to hold athletes from play because they report symptoms following injury, even if that is their individual "norm"? These questions highlight the need for a more individualized approach and

*Correspondence and reprint requests to: Garrett Thomas, 372 Moore Building, The Pennsylvania State University, University Park, PA, 16802, USA. E-mail: gat84@psu.edu understanding of how each athlete presents at baseline especially with regard to symptomatology. One common tool that is used to assess symptomatology is the Post-Concussion Symptom Scale (PCSS) from The Immediate PostConcussion Assessment and Cognitive Testing (ImPACT). The PCSS is a self-report measure that is often utilized at both baseline and post-concussion to capture an athlete's symptom profile. Despite its depiction as a "post-concussion" scale, previous research has demonstrated the utility of using the PCSS at baseline and has shown the value added in making comparisons to baseline evaluations following concussion. For example, Custer and colleagues (2016) found that athletes with high PCSS scores at baseline experienced greater decline in verbal and visual memory following concussion compared to those who reported no symptoms on the PCSS. This indicates that those reporting higher symptomatology at baseline may be at greater risk for negative outcomes following concussion than those who deny symptomatology. However, this begs the question: what 
leads an athlete to report symptoms consistent with concussion despite not experiencing an injury (i.e., at baseline)?

One possible factor associated with baseline symptom reporting is whether the athlete presents with significant affective symptomatology. Previous research has found that symptoms of stress, depression, and/or anxiety were positively correlated with post-concussion-like symptoms in individuals with no concussion history (Edmed \& Sullivan, 2012; Garden \& Sullivan, 2010). Similarly, Iverson (2005) demonstrated that patients who were clinically diagnosed with a depressive disorder demonstrated elevated symptom reporting on the British Columbia Post-concussion Symptom Inventory such that approximately $90 \%$ of patients in this sample met "liberal self-report criteria" for post-concussion syndrome (PCS) while over 50\% met conservative criteria for PCS despite not having sustained recent head injury. Another study found that individuals with depression but without recent mild traumatic brain injury (mTBI), reported significantly greater symptom scores on the British Columbia Post-concussion Symptom Inventory than those who had sustained mTBI but denied depression, though these groups both reported significantly fewer symptoms than those with both mTBI and depression (Lange et al., 2011). Relatedly, Kontos and colleagues (2012) demonstrated that, at 14 days postconcussion, depression was related to higher symptom scores. Previous work has also focused on symptom reporting in individuals with a history of mood disturbance. Despite the focus on history of disturbance rather than current complaint, these studies showed similar findings in that athletes with a history of mood disorder reported more symptoms with greater severity (Wallace et al., 2020; Weber et al., 2018), and that athletes with a history of psychiatric treatment were more likely to report emotional symptoms (Asken et al., 2017). Lastly, Snell and colleagues (2018) found that participants with chronic pain without history of mTBI also reported elevated post-concussion-like symptoms scores. Taken together, these findings suggest that there are many factors other than concussion that may lead to increased scores on self-report symptom measures.

Additionally, previous work by Riegler and colleagues (2019a) demonstrated the utility of using the PCSS affective factor as a screener for depression, suggesting that the affective symptoms captured on the PCSS demonstrate sensitivity similar to that of established depression screeners. Further, based on current understanding of depression and anxiety, these syndromes can lead to or exacerbate other difficulties such as cognitive difficulties (Castaneda et al., 2008), somatic and physical symptoms (Simms et al., 2012; Suls \& Howren, 2012), and/or sleep disturbances (Alvaro et al., 2013; Nyer et al., 2013). This understanding of the phenomenology of depression and anxiety is important because it may interfere with how the PCSS is interpreted given the overlap with potential concussion symptomatology. Specifically, a large number of symptoms of depression and anxiety overlap with items included in standard sports-concussion symptom checklists (e.g. sleep disturbance, mood changes, cognitive symptoms, and somatic symptoms) (Solomon et al.,
2015). This overlap may present challenges in discerning whether symptoms are pathognomonic of concussion or related to affective disturbance, which suggests that further research is warranted in order to improve clinical recommendations. Some of our previous work has focused on the association of affective symptomatology with neurocognitive performance (Guty \& Arnett, 2018; Riegler et al., 2020; Thomas, Guty, Riegler, \& Arnett, 2021); however, it is unclear how affective disturbances are associated with symptom reporting across the PCSS. One might expect athletes experiencing either depression or anxiety, or both together, to have an elevated affective cluster on the PCSS given the nature of such symptoms and overlap, but we are interested in understanding associations with other symptom clusters as well. Given that comorbidity has been associated with more severe symptoms and complaints (LeMoult, Castonguay, Joormann, \& McAleavey, 2013; Pratt et al., 2016), it is important to understand whether this is global or more circumscribed to the affective cluster. Thus, while the decision to evaluate symptom cluster scores in addition to total scores is predicated upon the limitations described above, the examination of the potential impact of elevated affective symptomatology on PCSS symptom cluster scores is largely exploratory, though it is possible that this approach may prove to be fruitful in helping to understand psychological influences on symptom reporting. Further, given research demonstrating that athletes tend to under-report symptomatology, particularly male athletes underreporting affective symptomatology and female athletes reporting more symptoms than male athletes at both baseline and following concussion, it is important to further explore patterns of symptom reporting (Asken et al., 2017; Covassin et al., 2006, 2012; McCrea et al., 2004; Meier et al., 2015; Moser et al., 2019).

\section{Current Study}

To summarize, the current study aims to fill gaps in the neuropsychology and sports-related concussion (SRC) literature regarding affective disturbance, particularly elevated concomitant anxiety/depressive symptoms, and its association with baseline symptom reporting in college athletes. While previous research has investigated various aspects of symptom reporting and affective disturbance, the current study is believed to be novel in that it is aimed at assessing symptom reporting, in total and by symptom cluster, in college athletes who report affective disturbance at the time of baseline testing. It is important to properly identify and document baseline symptomatology because it may allow for more appropriate comparisons following concussion. In other words, if an athlete reports elevated symptomatology at baseline, it may be less concerning if the same individual reports elevated symptomatology (within a comparable range) following concussion - especially as opposed to an athlete who denies symptomatology at baseline. Thus, it allows for providers to make decisions relative to the individual and may be used in conjunction with neuropsychological data. We hypothesize that: 1) athletes reporting affective 
disturbance (e.g., athletes in the depressive symptoms only, anxiety symptoms only, and concomitant groups) will report higher PCSS scores, indicating greater symptomatology, compared to healthy controls (HCs); and 2) athletes reporting elevated concomitant anxiety/depressive symptoms will report higher PCSS scores than athletes experiencing either depressive symptoms or anxiety symptoms alone.

\section{METHODS}

\section{Participants}

This study involved $833(\mathrm{M}=622, \mathrm{~F}=211)$ collegiate athletes who were involved in a concussion management program at an NCAA Division I University. The mean age was 18.51 years $(\mathrm{SD}=1.05)$ with a range from 17 to 24 . All participants completed a comprehensive neuropsychological test battery at baseline which included self-report measures of anxiety, depression, and the PCSS. Testing was completed as part of standard operating procedures within the athletic programs. Athletes from the following sports underwent baseline testing: football, men's and women's soccer, wrestling, men's and women's lacrosse, men's and women's ice hockey, men's and women's basketball, baseball, softball, crew, volleyball, and rugby. Athletes in the current study were selected from a larger group of athletes $(N=1050)$ receiving baseline testing between 2002 and 2019. Athletes were only included in the current study if they completed the PCSS, the Beck Depression Inventory-Fast Screen (BDI-FS) (Beck, Steer, \& Brown, 2000), and the NEO-Five Factor Inventory (NEO-FFI) (McCrae \& Costa, 2004) at baseline. All data were collected prior to the COVID-19 pandemic.

\section{Procedures}

This was a follow-up to previous work from a cross-sectional, retrospective research study conducted as part of the Sports Concussion Program at our NCAA Division I University (Thomas et al., 2021). Our Sports Concussion Program is based on the "Sports as Laboratory Assessment Model (SLAM)" (Bailey et al., 2010; Levin et al., 1989). All participants were referred for baseline testing by their athletic trainer or team physician and completed a 2.5-hour comprehensive neuropsychological test battery at baseline. The neuropsychological test battery was administered by undergraduate research assistants or graduate students who were supervised by a Ph.D.-level clinical neuropsychologist. Our study was conducted in compliance with our university's Institutional Review Board requirements and American Psychological Association ethical guidelines.

\section{Measures}

The BDI-FS was used to measure depressive symptoms in this study. The BDI-FS consists of seven items rated from
0 to 3 , with higher scores indicating more severe symptoms. The BDI-FS is well-validated for use in medical populations and demonstrates value in measuring depressive symptoms in concussed populations due to its ability to discriminate between depression symptoms and symptoms of a concussion (Riegler et al., 2019a). The distribution of the BDI-FS scores was not normal and demonstrated positive skewness and kurtosis in this sample. Further, a large percentage of individuals $(52.3 \%)$ scored 0 on the BDI-FS. Given these considerations, and in order to maintain consistency with the cutoffs for the NEO-FFI subscale (described below), the BDI-FS cutoffs were derived based on scores one standard deviation greater than the sample mean $(M=1.06$, $\mathrm{SD}=1.73$ ). As such, BDI-FS scores were dichotomized into two groups based on the presence of elevated depressive symptomatology $(\geq 3)$ or absence of elevated depressive symptomatology $(<3)$. These cutoffs were consistent for both males and females.

A modified subscale of the NEO-FFI was used to measure anxiety. Possible scores ranged from 0 to 16 with higher scores indicating more severe symptoms. The rationale and support for the development of this subscale can be found elsewhere (Thomas et al., 2021). For purposes of this paper, it is important to note that the NEO-FFI Anxiety Subscale has been shown to correlate with the Anxiety Sensitivity Index (ASI: Reiss, Peterson, Gursky, \& McNally, 1986) at levels comparable to the ASI's correlation with other trait anxiety measures (McNally \& Lorenz, 1987; Reiss et al., 1986; Sandin et al., 2001; Thomas et al., 2021). The ASI has also shown reliability in assessing anxiety in college students (Peterson \& Heilbronner, 1987). Thus, the ASI appears to be an appropriate comparison for validation, and the NEOFFI Anxiety Subscale can be considered an acceptable measure of anxiety for the current study.

Given that there is not a validated cutoff for the NEO-FFI Anxiety subscale, the current study used a score of one standard deviation above the sample mean $(M=6.51$, $\mathrm{SD}=2.84)$ to indicate clinical significance. As such, NEO-FFI Anxiety subscale scores were dichotomized into two groups based on the presence of elevated anxiety symptomatology $(\geq 10)$ or absence of elevated anxiety symptomatology $(<10)$ which is comparable to cutoffs used in previous work (Thomas et al., 2021). These cutoffs were consistent for both males and females. Of note, the NEO-FFI Anxiety subscale scores were normally distributed. Rationale for using one standard deviation above the mean cutoffs, in addition to dichotomizing presence or absence of depression and/or anxiety, is predicated upon our interest in exploring the role of elevated affective symptomatology rather than clinical diagnoses. Moreover, we are specifically interested in evaluating differences between groups, particularly the concomitant depression/ anxiety group compared to the other groups. Further, using a cutoff of one standard deviation is a commonly used method to indicate impairment (Lezak et al., 2004), and maintains symmetry with the approach utilized in our previous work (Thomas et al., 2021). 
Table 1. Composition of groups defined by self-report symptoms of depression and anxiety

\begin{tabular}{|c|c|c|c|c|}
\hline & \multicolumn{2}{|c|}{ Sex } & \multirow[b]{2}{*}{$N^{1}$} & \multirow{2}{*}{$\begin{array}{l}\% \text { of Sample } \\
\text { Population }\end{array}$} \\
\hline & Male & Female & & \\
\hline Baseline & 622 & 211 & 833 & \\
\hline Healthy Control & 455 & 126 & 581 & 69.7 \\
\hline Depressive Symptoms Only & 102 & 34 & 136 & 16.3 \\
\hline Anxiety Symptoms Only & 29 & 25 & 54 & 6.5 \\
\hline $\begin{array}{l}\text { Concomitant Depressive and } \\
\text { Anxiety Symptoms }\end{array}$ & 36 & 26 & 62 & 7.5 \\
\hline
\end{tabular}

${ }^{1}$ These groups are all independent, meaning that athletes captured in the Depression Only or Anxiety Only groups are not counted toward the Concomitant Group. In doing so, we attempted to make the groups as diagnostically pure as possible. For example, the $n$ of the Anxiety Only group is smaller than the Concomitant group because many of the athletes experiencing symptoms of anxiety also experience symptoms of depression, thus falling into the Concomitant group and not the Anxiety Only group.

The PCSS is a self-report symptom measure that is comprised of 22 items describing common post-concussion symptoms on a scale of 0 to 6 with 0 being 'None' and 6 being 'Severe' (Lovell et al., 2006). While much of previous research has focused on the PCSS Total Score when evaluating symptomatology at baseline and/or post-concussion (Covassin et al., 2012; McClincy et al., 2006), this approach may not be sufficient as it does not account for the athlete's specific symptom profile. Thus, this approach may not be an accurate representation of the athlete's overall symptomatology. Therefore, we decided to evaluate both PCSS Total Scores, as well as specific symptom clusters. To examine the latter, PCSS scores were broken down into 4 symptom clusters based on previous factor analytic work demonstrating that the PCSS items load onto 4 primary factors (e.g., Cognitive, Physical, Affective, and Sleep) with headache as a separate $5^{\text {th }}$ factor (Merritt et al., 2015). Headache was also included as a separate factor because previous work has shown that headaches are one of the most common and persistent symptoms associated with sports-related concussion (Heyer et al., 2016; Kontos et al., 2013; Womble et al., 2019) and may predict prolonged recovery following concussion (Iverson et al., 2017). This inclusion of headache as a separate factor, in addition to clusters of Cognitive, Physical, Affective, and Sleep symptoms, is supported by previous research (Guty \& Arnett, 2018; Merritt et al., 2015; Riegler et al., 2021). These symptom clusters and their associated PCSS items are described in Table 1. Of note, the symptom clusters differ in the associated number of items, thus leading to different possible maximum scores.

\section{Quantitative Approach}

Athletes were then separated into four groups (HC $(n=581)$, Depression Only $(n=136)$, Anxiety Only $(n=54)$, and Concomitant Depression/Anxiety $(n=62)$ ) based on their anxiety and depression scores (see Table 2). Of note, the groups were unequal in size and some of the groups differed
Table 2. PCSS symptom clusters and associated items

\begin{tabular}{lcll}
\hline \hline Cognitive & Physical & Affective & Sleep \\
\hline $\begin{array}{c}\text { Feeling slowed } \\
\text { down }\end{array}$ & Nausea & Irritability & Fatigue \\
$\begin{array}{c}\text { Feeling men- } \\
\text { tally "foggy" }\end{array}$ & Vomiting & Sadness & $\begin{array}{c}\text { Trouble Falling } \\
\text { Asleep }\end{array}$ \\
$\begin{array}{c}\text { Difficulty } \\
\text { Concentrating }\end{array}$ & Dizziness & Nervousness & $\begin{array}{c}\text { Sleeping Less } \\
\text { Than Usual }\end{array}$ \\
$\begin{array}{c}\text { Difficulty } \\
\text { Remembering }\end{array}$ & $\begin{array}{c}\text { Balance } \\
\text { problems }\end{array}$ & Feeling More & Drowsiness \\
& $\begin{array}{c}\text { Sensitivity } \\
\text { to Light }\end{array}$ & & \\
& Sensitivity \\
to Noise & & \\
& Visual & \\
& Problems & \\
\hline \hline
\end{tabular}

Table adapted from Merritt, V.C., \& Arnett, P.A. (2014). Premorbid predictors of post-concussion symptoms in collegiate athletes. Journal of Clinical \& Experimental Neuropsychology, 36(10), 1098-1111; Table 5, p. 1105.

in the proportion of male to female athletes, an issue that will be discussed in subsequent sections. The groups did not significantly differ in terms of mean age or proportion of athletes who reported previous concussion(s).

We first conducted linear regressions to first examine the effect of Group (i.e., affective symptomatology presentation) on Total PCSS Scores. We employed a dummy coding approach so we could make meaningful comparisons between $\mathrm{HCs}$ and each of the affective groups separately while maximizing statistical power. Next, in line with previous research aimed at exploring PCSS Symptom Cluster Scores instead of PCSS Total Score (Merritt et al., 2014), we conducted a series of six multivariate analyses of variance (MANOVAs) comparing each of the affective groups to the healthy control group, as well as to the other affective groups. A Bonferroni correction was calculated to control for multiple comparisons (i.e., the six multivariate tests). As such, a $p$-value of .008 was used to indicate statistical significance for the multivariate tests. The univariate tests were not subject to correction given that utilizing a MANOVA framework effectively controls for multiple comparisons when evaluating univariate effects.

\section{RESULTS}

\section{PCSS Total Scores}

Overall, results revealed a significant effect of Group on Total PCSS Score, $F(3,829)=45.38, p<.001, \eta_{p}{ }^{2}=.14 .{ }^{1}$ We found that, compared to HCs, the concomitant group reported significantly greater Total PCSS Scores, $t(829)=11.25, p<.001$, $d=1.02,{ }^{2}$ as did the depressive symptoms only group, $t(831)=4.58, p<.001, d=.45$. The anxiety symptoms only

\footnotetext{
${ }^{1}$ Per Cohen (2013), effect sizes for $\eta_{\mathrm{p}}{ }^{2}$ are as follows: .01 to $.05=$ small; $.06-$ $.14=$ medium; $>.14=$ large

${ }^{2}$ Per Cohen (2013), effect size cutoffs for $d$ are as follows: $0.2=$ small; $0.5=$ medium; $0.8=$ large.
} 


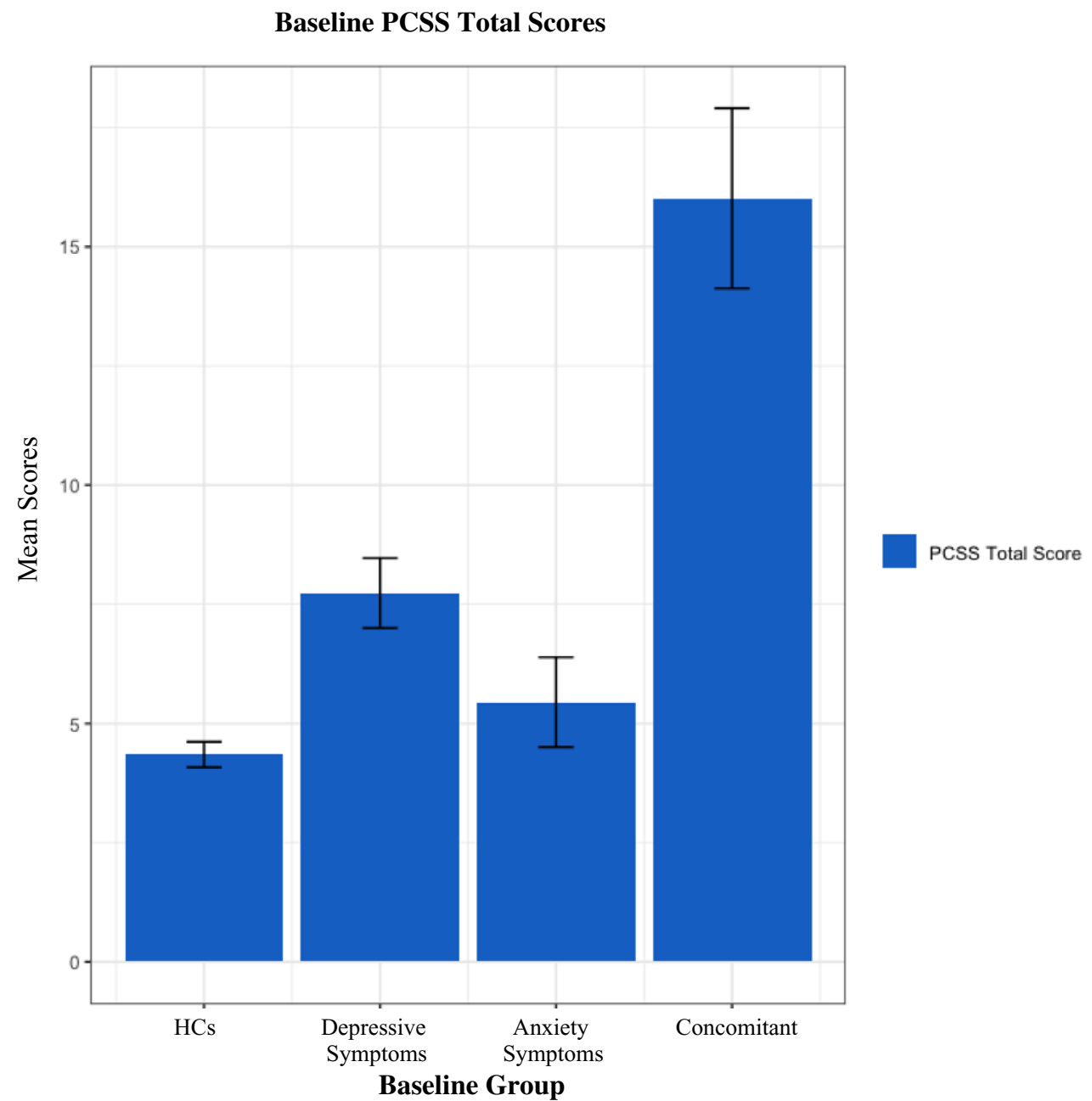

Fig. 1. Baseline PCSS total scores by group.

group did not show a significant difference in Total PCSS Score compared to HCs, $t(829)=.99, p=.32, d=.18$. Further, we found that the concomitant group reported significantly greater Total PCSS Scores than both the depressive symptoms only group, $t(829)=-6.96, p<.001, d=.68$, and the anxiety symptoms only group, $t(829)=-7.32, p<.001$, $d=.91$. The depressive symptoms only group showed marginally elevated Total PCSS Score compared to the anxiety symptoms only group, $t(829)=-1.83, p=.07, d=.30$. Total PCSS Score results are depicted in Figure 1. To further understand these findings, we conducted additional analyses aimed at exploring potential differences in symptom cluster scores. PCSS Total and Symptom Cluster Score means, standard deviation, and score ranges are presented in Table 3.

\section{PCSS Symptom Cluster Scores}

In line with previous research by Merritt et al. (2014), we conducted additional analyses, which were planned a priori, to explore the presentation of symptomatology broken down into 5 symptom clusters: Cognitive, Physical, Affective, Sleep, and Headache. Results are depicted in Figure 2.

\section{Affective groups compared to healthy controls}

MANOVA results revealed that, overall, all three affective groups reported significantly greater symptomatology than HCs, with the concomitant group showing the largest difference compared to HCs. These results are further explored in subsequent sections.

Concomitant group vs healthy controls. Overall, the concomitant group reported significantly greater symptomatology than the $\mathrm{HC}$ group, $F(5,637)=44.09, p<.001, \eta_{p}{ }^{2}=.26$. Further, while all symptom clusters were significantly elevated in the concomitant group, the factors with the largest effects relative to HCs were the affective cluster, $F(1,641)=$ 211.03, $p<.001, \eta_{p}{ }^{2}=.25$, cognitive cluster, $F(1,641)=$ 81.32, $p<.001, \eta_{p}{ }^{2}=.11$, and sleep cluster, $F(1,641)=42.45, p<.001, \eta_{p}{ }^{2}=.06($ see Table 4$)$.

Depressive symptoms only group vs healthy controls. Overall, the depressive symptoms only group reported significantly greater symptomatology than the $\mathrm{HC}$ group, $F(5,711)=$ $7.49, p<.001, \eta_{p}^{2}=.05$. Further, the depressive symptoms only group reported significantly elevated symptomatology, 
Table 3. PCSS symptom cluster means, SDs and score ranges

\begin{tabular}{lcccc}
\hline \hline & HCs $[\boldsymbol{M}(\mathbf{S D})]$ & Comorbid $[\boldsymbol{M}(\mathbf{S D})]$ & Depression $[\boldsymbol{M}$ (SD)] & Anxiety $[\boldsymbol{M}(\mathbf{S D})]$ \\
\hline Symptom Cluster (score range) & & & \\
Total Score (0-120) & $4.35(6.44)$ & $16.02(14.87)$ & $1.74(8.56)$ & $5.44(6.92)$ \\
Cognitive (0-24) & $.74(1.78)$ & $3.31(4.18)$ & $.67(1.69)$ & $1.59(3.03)$ \\
Physical (0-42) & $.35(1.54)$ & $1.65(3.30)$ & $1.87(2.94)$ & $.33(.78)$ \\
Affective (0-24) & $.88(1.76)$ & $5.32(5.08)$ & $2.98(3.43)$ & $1.15(1.76)$ \\
Sleep (0-24) & $1.90(2.64)$ & $4.34(4.04)$ & $.42(.87)$ & $.35(.81)$ \\
Headache (0-6) & $.33(.86)$ & $.82(1.14)$ & & \\
\hline \hline
\end{tabular}

Baseline PCSS Symptom Cluster Scores

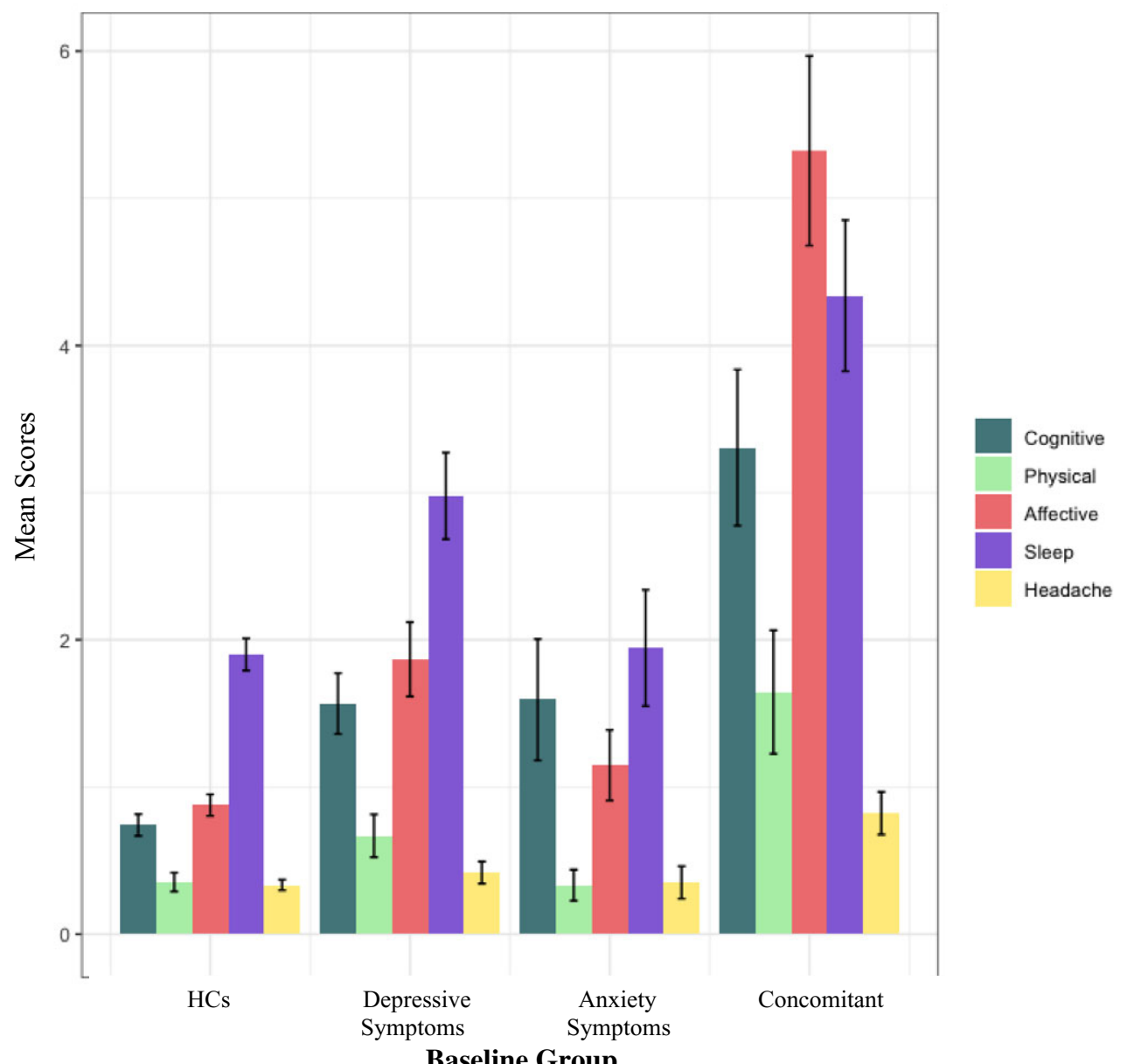

Baseline Group

Fig. 2. Baseline PCSS symptom cluster scores by group.

compared to HCs, on every symptom cluster except headache. The factors with the largest effects were the affective cluster, $F(1,715)=26.10, p<.001, \eta_{p}{ }^{2}=.04$, cognitive cluster, $F(1,715)=20.42, p<.001, \eta_{p}{ }^{2}=.03$, and sleep cluster, $F(1,715)=16.28, p<.001, \eta_{p}^{2}=.02($ see Table 4$)$.

Anxiety symptoms only group vs healthy controls. Overall, the anxiety symptoms only group reported significantly greater symptomatology than the $\mathrm{HC}$ group, $F(5,629)=$ $7.49, p=.003, \eta_{p}{ }^{2}=.03$. However, the anxiety symptoms only group reported significantly increased symptomatology, compared to $\mathrm{HCs}$, on only the cognitive symptoms cluster, $F(1,633)=9.73, p=.002, \eta_{p}^{2}=.02$. Interestingly, the anxiety symptoms only group did not differ from HCs in affective symptomatology, $F(1,633)=1.17, p=.28, \eta_{p}{ }^{2}=.002($ see Table 4$)$.

Affective groups compared to affective groups (depressive vs. anxiety vs. concomitant symptoms)

Depressive symptoms only group vs anxiety symptoms only group. Overall, the depressive symptoms only group did 
Table 4. PCSS symptom clusters: affective groups compared to healthy controls

\begin{tabular}{lrll}
\hline \hline & $\mathrm{F}$ & $\boldsymbol{p}$-value & Partial eta squared $\left(\boldsymbol{\eta}^{\mathbf{2}}\right)^{\mathbf{a}}$ \\
\hline Concomitant vs. Healthy Control (HC) & \\
Overall & 44.09 & $<.001^{* * *}$ & .26 \\
Cognitive & 81.32 & $<.001^{* * *}$ & .11 \\
Physical & 29.33 & $<.001^{* * *}$ & .04 \\
Affective & 211.03 & $<.001^{* * *}$ & .25 \\
Sleep & 42.45 & $<.001^{* * *}$ & .06 \\
Headache & 16.98 & $<.001^{* * *}$ & .03 \\
Anxiety Symptoms vs. HC & \\
Overall & 3.60 & $.003^{* *}$ & .03 \\
Cognitive & 9.73 & $.002^{* *}$ & .02 \\
Physical & .01 & .92 & .00 \\
Affective & 1.17 & .28 & .002 \\
Sleep & .01 & $.91^{*}$ & .00 \\
Headache & .02 & .89 & .00 \\
Depressive Symptoms vs. HC & .05 \\
Overall & 7.49 & $<.001^{* * *}$ & .03 \\
Cognitive & 20.42 & $<.001^{* * *}$ & .006 \\
Physical & 4.43 & $.04^{* *}$ & .04 \\
Affective & 26.10 & $<.001^{* * *}$ & .02 \\
Sleep & 16.28 & $<.001^{* * *}$ & .001 \\
Headache & 1.06 & .30 & \\
\hline \hline
\end{tabular}

a partial eta squared effect sizes: small (.01 - .05); medium (.06 - .14); large $(>.14)$.

*marginally significant at the .10 level.

** significant at the .05 level.

*** significant at the .001 level.

not differ significantly from the anxiety symptoms only group, though there was a small effect size, $F(5,184)=1.85, p=.11, \eta_{p}{ }^{2}=.05$. Of note, the depressive symptoms only group showed a marginal increase in sleep symptoms, $F(1,188)=3.81, p=.052, \eta_{p}{ }^{2}=.02$, and affective symptoms, $F(1,188)=2.82, p=.095, \eta_{p}{ }^{2}=.02$, compared to the anxiety symptoms only group. These groups did not differ on any of the other symptom clusters (see Table 5).

Concomitant group vs. depressive symptoms only group. Overall, the concomitant group reported significantly greater symptomatology than the depressive symptoms only group, $F(5,192)=7.55, p<.001, \eta_{p}{ }^{2}=.16$. Further, while all symptom clusters were significantly elevated in the concomitant group, the factors with the largest effects relative to the depressive symptoms only group were the affective cluster, $F(1,196)=36.32, p<.001, \eta_{p}{ }^{2}=.16$, and cognitive cluster, $F(1,196)=13.68, p<.001, \eta_{p}^{2}=.07$ (see Table 5).

Concomitant group vs anxiety symptoms only group. Overall, the concomitant group reported significantly greater symptomatology than the anxiety symptoms only group, $F(5,110)=8.18, p<.001, \eta_{p}{ }^{2}=.27$. Further, while all symptom clusters were significantly elevated in the concomitant group, the factors with the largest effects relative to the anxiety symptoms only group were the affective cluster,
Table 5. PCSS symptom clusters: affective groups compared to affective groups

\begin{tabular}{lccc}
\hline \hline & $\mathrm{F}$ & $\boldsymbol{p}$-value & Partial eta squared $\left(\boldsymbol{\eta}^{2) \mathbf{a}}\right.$ \\
\hline Depressive vs. & Anxiety & Symptoms & \\
Overall & 1.85 & .11 & .05 \\
Cognitive & .004 & .95 & .00 \\
Physical & 1.96 & .16 & .01 \\
Affective & 2.82 & $.095^{*}$ & .02 \\
Sleep & 3.81 & $.052^{*}$ & .02 \\
Headache & .24 & .63 & .001 \\
Concomitant vs. Anxiety & Symptoms & \\
Overall & 8.18 & $<.001^{* * *}$ & .27 \\
Cognitive & 6.23 & $.01^{* *}$ & .05 \\
Physical & 8.11 & $.005^{* *}$ & .07 \\
Affective & 32.98 & $<.001^{* * * *}$ & .22 \\
Sleep & 13.06 & $<.001^{* * *}$ & .10 \\
Headache & 6.43 & $.01^{* *}$ & .05 \\
Concomitant vs. Depressive Symptoms & \\
Overall & 7.55 & $<.001^{* * *}$ & .16 \\
Cognitive & 13.68 & $<.001^{* * *}$ & .07 \\
Physical & 7.56 & $.01^{* *}$ & .04 \\
Affective & 36.32 & $<.001^{* * * *}$ & .16 \\
Sleep & 5.98 & $.02^{* *}$ & .03 \\
Headache & 7.46 & $.01^{* * *}$ & .04 \\
\hline \hline
\end{tabular}

${ }^{a}$ partial eta squared effect sizes: small (.01 - .05); medium (.06 - .14); large (>.14).

*marginally significant at the .10 level.

** significant at the .05 level.

$* * *$ significant at the .001 level.

$F(1,114)=32.98, p<.001, \eta_{p}^{2}=.22$, sleep cluster, $F(1,114)=13.06, p<.001, \eta_{p}{ }^{2}=.10$, and physical cluster, $F(1,114)=8.11, p=.005, \eta_{p}^{2}=.07$ (see Table 5).

\section{DISCUSSION}

The current paper examined the relationship between significant affective symptomatology and self-report symptomatology across 5 symptom clusters on the PCSS, as well as total number of symptoms. Some of our previous work has found that those experiencing significant affective symptomatology show greater neurocognitive impairments at baseline (Guty \& Arnett, 2018; Riegler et al., 2020; Thomas et al., 2021), and previous research has also explored aspects of the relationship between affective disturbance (particularly depression) and symptom reporting (Asken et al., 2017; Garden \& Sullivan, 2010; Iverson et al., 2017; Wallace et al., 2020; Weber et al., 2018). However, additional research is warranted to better understand how an athlete's affective state, particularly when reporting elevated concomitant depressive/anxiety symptoms, is associated with specific symptom clusters at baseline.

Our findings suggest that athletes experiencing elevated concomitant depressive/anxiety symptoms reported significantly greater levels of symptomatology across all 5 clusters of the PCSS compared to HCs. Further, our findings demonstrate that athletes experiencing elevated 
concomitant affective symptoms tend to report greater symptomatology than those with elevated affective symptoms in only one domain (e.g., anxiety or depressive symptoms alone). Thus, it appears that the presentation of two co-occurring affective disturbances negatively impacts athletes over and above the presentation of only one affective disturbance. While the current study examined athletes with elevated symptomatology rather than those with clinical disorders, these findings are inline with previous research showing that the burden of disease increases with each additional diagnosis, and that those with comorbid psychological disorders typically demonstrate poorer functioning and less responsiveness to treatment compared to those with only one psychological disturbance (Emmanuel et al., 1998; Gadermann et al., 2012; Walczak et al., 2018). This finding is important as concomitant affective disturbance, and its effects on athletes, has remained relatively unstudied to this point yet clearly plays an important role in athletes' functioning.

Unsurprisingly, the effect size for the affective cluster was largest for the concomitant and depressive symptoms only groups, but it is important to recognize the cognitive symptom cluster was next largest. This latter finding supports previous research findings that show that those experiencing depression or concomitant affective disturbance perform significantly worse than HCs on objective neuropsychological measures (Riegler et al., 2019b; Thomas et al., 2021). Considering the widespread use of objective neuropsychological testing in determining recovery from concussion, exploring how factors other than concussion, like affective symptomatology, may skew cognitive performance is critical. Moreover, it is also important to highlight that athletes in the concomitant group report significantly greater headache symptomatology - something that is deemed to be a persistent and "hallmark" symptom of concussion. Thus, it is important to take this into account when making postconcussion comparisons as this presentation may not necessarily be pathognomonic of concussion. Relatedly, it is critical to note that the sleep cluster has the highest score for the healthy control and anxiety groups, and the second highest score for the depression and concomitant depression/anxiety groups. These results support previous findings regarding the negative impact of sleep disturbance on college athletes (Riegler et al., 2021), and also consistent with prior work suggesting that sleep disturbance is a widespread problem for all athletes (Asken et al., 2016, 2017; Iverson et al., 2015). Considering that sleep may be easily modifiable, it may be an important point of clinical intervention. It is also important to note that the physical symptoms cluster showed relatively low scores across groups, despite possessing the potential for the greatest score due to the cluster containing 7 items. In contrast, the cognitive, affective and sleep clusters contained only 4 items, and the headache cluster only contained one item. Thus, it appears that very few people reported physical symptoms at baseline, which suggests the physical symptoms cluster may be more specific to concussion itself rather than secondary factors like depression, anxiety, or comorbidity.
Interestingly, the anxiety symptoms only group showed similar elevations on the cognitive symptom cluster, but this group did not show a significant increase in affective symptoms compared to HCs. One possible explanation is predicated upon the types of questions that make up the affective cluster of the PCSS, as well as our use of the NEO-FFI Anxiety Subscale to define the anxiety symptoms only group. Given that anxiety and depression often overlap, we attempted to obtain a pure anxiety measure by creating an Anxiety Subscale from the Neuroticism Facet of the NEOFFI. The development of this subscale is described elsewhere (Thomas et al., 2021), but it is important to note that the PCSS affective symptom cluster is comprised of 4 items, 3 of which phenomenologically describe depression and only 1 of which appears related to anxiety. Considering the Anxiety Subscale is designed to be a purer measure of anxiety that does not include depressive symptoms, it is perhaps not surprising that there was not as much overlap as one might have expected. Further, we believe this finding actually gives credence to using the NEO-FFI Anxiety Subscale as an anxiety measure, as it did not correlate with BDI-FS total score in our sample population, $r(847)^{3}=.01, p=.75$.

Despite this possible support for using the NEO-FFI Anxiety Subscale as a relatively pure anxiety measure, this could also be considered a limitation of the study in that we did not use a stand-alone anxiety measure. While this anxiety subscale has been shown to correlate with the ASI at a level comparable to other anxiety measures as previously described, the scale has not been subject to rigorous validation. Another possible limitation is that, because we created the affective groups based on cutoff scores derived from the sample mean and standard deviation, rather than clinical cutoffs, we are capturing those who are experiencing affective symptomatology rather than true clinical disorders. However, we believe these cutoffs are still of utility given that they are derived from base rates within our sample, and also because of some athletes' tendency to underreport symptoms (McCrea et al., 2004; Meier et al., 2015). Further, these findings demonstrate that, even if athletes do not meet full criteria for a clinical disorder, they may still be prone to reporting greater symptomatology which is of clinical significance. Another possible limitation is that the groups differed in the proportion of male to female athletes, such that the Anxiety and Concomitant groups had a significantly larger proportion of females to males than the $\mathrm{HC}$ and Depression groups. Given that previous research has demonstrated that female athletes tend to report more symptoms than males, both at baseline and post-concussion (Asken et al., 2017; Covassin et al., 2012), it is plausible that the results are influenced by potential sex differences. For example, if the groups were evenly distributed in terms of sex, it is possible that the $\mathrm{HC}$ and Depression groups would report higher PCSS scores, or the Anxiety and Concomitant groups would report lower PCSS scores, which could lead

\footnotetext{
${ }^{3}$ The degrees of freedom listed are larger than previously reported in our analyses. This is because we included all athletes who completed the BDI-FS and NEO-FFI for this correlation, we and did not exclude those who did not have complete PCSS data.
} 
to less significant differences between groups. Alternatively, if our sample included more female athletes, it is possible that BDI-FS cutoff scores would differ between males and females, thus requiring a different set of cutoffs. Similar issues have been discussed, with proposed solutions, in Arnett et al. (2016). Lastly, this study is also limited in that we are not able to fully account for potential secondary influences, such as history of migraine, vestibular disorders, or medication usage, which may also influence symptom reporting. Further, given that we do not require athletes to report all medical and/or psychiatric history at the time of testing, there may be factors other than depression and anxiety that contribute to these findings.

In sum, the findings of this study are important because they demonstrate the impact of concomitant depressive, and, to a lesser extent, anxiety symptoms on PCSS symptom reporting - both in total and by symptom cluster. These relationships are important to understand given the emphasis placed on symptom reporting in concussion management and when making return-to-activity decisions. For example, despite not currently experiencing a concussion, the concomitant group demonstrated significantly elevated symptomatology on the PCSS, which could skew post-concussion comparisons if the athlete's affective problems persist. Alternatively, if an athlete experiences concomitant depression and anxiety at baseline, but not post-concussion, symptoms that may actually result from concussion may be masked by their elevated symptom profile at baseline. Thus, it is recommended that athletes are routinely screened for mood disturbances using brief screeners, like the NEO-FFI Anxiety Subscale, BDI-FS, and/or the Affective Scale from the PCSS (Riegler et al., 2019a), so that athletes who might be at risk for affective symptomatology can then be referred for a more thorough assessment and provided resources if necessary. Further, by routinely screening for affective symptoms at baseline, this allows providers to be aware that these symptoms are present and thus able to account for the potential influence of these symptoms, as well as potential differences in symptom reporting, during subsequent testing. Future research may be focused on incorporating the influence of affective symptoms in RTL and/or RTP decision-making algorithms similar to the algorithm present by Arnett and colleagues (2016). Taken with previous findings (Thomas et al., 2021), it is evident that people with concomitant affective disturbance have cognitive difficulties. However, without awareness of such difficulties at baseline, it could be assumed that cognitive difficulties associated with injury are related to concussion rather than underlying affective disturbance. Thus, it is vital that providers are aware of athlete's baseline functioning when making clearance decisions.

Lastly, it is also important to understand potential discrepancies in symptom reporting because symptom reduction plays a large role in determining whether an athlete is allowed to return to play or return to learn following concussion. Considering the important role of academics in the lives of student-athletes, it is critical that pre-existing mood disorders not interfere with return to learning after concussion and that return to learning should always precede unrestricted return to play. Further, considering that classroom performance may be impacted more by some symptom clusters over others (e.g., cognitive symptoms), it is important to gain a more nuanced understanding of an athlete's symptom profile - particularly in their day-to-day functioning prior to sustaining concussion in order to make more thoughtful clinical decisions after concussion.

\section{FINANCIAL SUPPORT}

The authors do not have a financial investment in this study or the outcome of the data to report.

\section{CONFLICT OF INTEREST}

The authors do not have conflicts of interest.

\section{ETHICAL STANDARDS}

Our study was conducted in compliance with our university's Institutional Review Board requirements and American Psychological Association ethical guidelines.

\section{REFERENCES}

Alvaro, P.K., Roberts, R.M., \& Harris, J.K. (2013). A systematic review assessing bidirectionality between sleep disturbances, anxiety, and depression. Sleep, 36(7), 1059-1068. doi: 10.5665/ sleep. 2810.

Arnett, P., Meyer, J., Merritt, V., \& Guty, E. (2016). Neuropsychological testing in mild traumatic brain injury: What to do when baseline testing is not available. Sports Medicine and Arthroscopy Review, 24(3), 116-122. doi: 10. 1097/JSA.0000000000000123.

Asken, B.M., McCrea, M.A., Clugston, J.R., Snyder, A.R., Houck, Z.M., \& Bauer, R.M. (2016). "Playing through it": Delayed reporting and removal from athletic activity after concussion predicts prolonged recovery. Journal of Athletic Training, 51(4), 329-335. doi: 10.4085/1062-6050-51.5.02.

Asken, B.M., Snyder, A.R., Clugston, J.R., Gaynor, L.S., Sullan, M.J., \& Bauer, R.M. (2017). Concussion-like symptom reporting in non-concussed collegiate athletes. Archives of Clinical Neuropsychology, 32(8), 963-971. doi: 10.1093/arclin/acx018.

Bailey, C.M., Samples, H.L., Broshek, D.K., Freeman, J.R., \& Barth, J.T. (2010). The relationship between psychological distress and baseline sports-related concussion testing. Clinical Journal of Sport Medicine: Official Journal of the Canadian Academy of Sport Medicine, 20(4), 272-277. doi: 10.1097/ JSM.0b013e3181e8f8d8.

Beck, A., Steer, R., \& Brown, G. (2000). BDI-Fast Screen for Medical Patients Manual. San Antonio, TX: Psychological Corporation.

Castaneda, A.E., Tuulio-Henriksson, A., Marttunen, M., Suvisaari, J., \& Lönnqvist, J. (2008). A review on cognitive impairments in depressive and anxiety disorders with a focus on young adults. 
Journal of Affective Disorders, 106(1), 1-27. doi: 10.1016/j.jad. 2007.06.006.

Covassin, T., Elbin, R.J., Harris, W., Parker, T., \& Kontos, A. (2012). The role of age and sex in symptoms, neurocognitive performance, and postural stability in athletes after concussion. The American Journal of Sports Medicine, 40(6), 1303-1312. doi: 10. 1177/0363546512444554.

Covassin, T., Swanik, C.B., Sachs, M., Kendrick, Z., Schatz, P., Zillmer, E., \& Kaminaris, C. (2006). Sex differences in baseline neuropsychological function and concussion symptoms of collegiate athletes. British Journal of Sports Medicine, 40(11), 923-927. doi: 10.1136/bjsm.2006.029496.

Custer, A., Sufrinko, A., Elbin, R.J., Covassin, T., Collins, M., \& Kontos, A. (2016). High baseline postconcussion symptom scores and concussion outcomes in athletes. Journal of Athletic Training, 51(2), 136-141. doi: 10.4085/1062-6050-51.2.12.

Edmed, S., \& Sullivan, K. (2012). Depression, anxiety, and stress as predictors of postconcussion-like symptoms in a non-clinical sample. Psychiatry Research, 200(1), 41-45. doi: 10.1016/j. psychres.2012.05.022.

Emmanuel, J., Simmonds, S., \& Tyrer, P. (1998). Systematic review of the outcome of anxiety and depressive disorders. The British Journal of Psychiatry, 173(S34), 35-41. doi: 10.1192/ S0007125000293501.

Gadermann, A.M., Alonso, J., Vilagut, G., Zaslavsky, A.M., \& Kessler, R.C. (2012). Comorbidity and disease burden in the National Comorbidity Survey Replication (NCS-R). Depression and Anxiety, 29(9), 797-806. doi: 10.1002/da.21924.

Garden, N. \& Sullivan, K.A. (2010). An examination of the base rates of post-concussion symptoms: The influence of demographics and depression. Applied Neuropsychology, 17(1), 1-7. doi: 10.1080/09084280903297495.

Guty, E. \& Arnett, P. (2018). Post-concussion symptom factors and neuropsychological outcomes in collegiate athletes. Journal of the International Neuropsychological Society: JINS, 24(7), 684-692. doi: 10.1017/S135561771800036X.

Heyer, G.L., Young, J.A., Rose, S.C., McNally, K.A., \& Fischer, A.N. (2016). Post-traumatic headaches correlate with migraine symptoms in youth with concussion. Cephalalgia: An International Journal of Headache, 36(4), 309-316. doi: 10. $1177 / 0333102415590240$.

Iverson, G.L. (2005). Outcome from mild traumatic brain injury. Current Opinion in Psychiatry, 18(3), 301. doi: 10.1097/01. yco.0000165601.29047.ae.

Iverson, G.L., Gardner, A.J., Terry, D.P., Ponsford, J.L., Sills, A.K., Broshek, D.K., \& Solomon, G.S. (2017). Predictors of clinical recovery from concussion: A systematic review. British Journal of Sports Medicine, 51(12), 941-948. doi: 10.1136/ bjsports-2017-097729.

Iverson, G.L., Silverberg, N.D., Mannix, R., Maxwell, B.A., Atkins, J.E., Zafonte, R., \& Berkner, P.D. (2015). Factors associated with concussion-like symptom reporting in high school athletes. JAMA Pediatrics, 169(12), 1132-1140. doi: 10.1001/jamapediatrics. 2015.2374.

Kontos, A.P., Covassin, T., Elbin, R.J., \& Parker, T. (2012). Depression and neurocognitive performance after concussion among male and female high school and collegiate athletes. Archives of Physical Medicine and Rehabilitation, 93(10), 1751-1756. doi: 10.1016/j.apmr.2012.03.032.

Kontos, A.P., Elbin, R.J., Lau, B., Simensky, S., Freund, B., French, J., \& Collins, M.W. (2013). Posttraumatic migraine as a predictor of recovery and cognitive impairment after sport-related concussion. The American Journal of Sports Medicine, 41(7), 1497-1504. doi: 10.1177/0363546513488751.

Lange, R.T., Iverson, G.L., \& Rose, A. (2011). Depression strongly influences postconcussion symptom reporting following mild traumatic brain injury. The Journal of Head Trauma Rehabilitation, 26(2), 127-137. doi: 10.1097/HTR. 0b013e3181e4622a.

LeMoult, J., Castonguay, L.G., Joormann, J., \& McAleavey, A. (2013). Depression. In L.G. Castonguay \& T.F. Oltmanns (Eds.), Psychopathology: From science to clinical practice, (pp. 17-61). New York, NY: The Guilford Press.

Levin, H.S., Eisenberg, H.M., \& Benton, A.L. (Eds.). (1989). Mild Head Injury. USA: Oxford University Press.

Lezak, M.D., Howieson, D.B., Loring, D.W., \& Fischer, J.S. (2004). Neuropsychological Assessment. USA: Oxford University Press.

Lovell, M.R., Iverson, G.L., Collins, M.W., Podell, K., Johnston, K.M., Pardini, D., Pardini, J., Norwig, J., \& Maroon, J.C. (2006). Measurement of symptoms following sports-related concussion: Reliability and normative data for the postconcussion scale. Applied Neuropsychology, 13(3), 166174. doi: 10.1207/s15324826an1303_4.

McClincy, M.P., Lovell, M.R., Pardini, J., Collins, M.W., \& Spore, M.K. (2006). Recovery from sports concussion in high school and collegiate athletes. Brain Injury, 20(1), 33-39. doi: 10.1080/ 02699050500309817.

McCrae, R.R. \& Costa, P.T. (2004). A contemplated revision of the NEO Five-Factor Inventory. Personality and Individual Differences, 36(3), 587-596. doi: 10.1016/S0191-8869(03) 00118-1.

McCrea, M., Hammeke, T., Olsen, G., Leo, P., \& Guskiewicz, K. (2004). Unreported concussion in high school football players: Implications for prevention. Clinical Journal of Sport Medicine: Official Journal of the Canadian Academy of Sport Medicine, 14(1), 13-17.

McNally, R.J. \& Lorenz, M. (1987). Anxiety sensitivity in agoraphobics. Journal of Behavior Therapy and Experimental Psychiatry, 18(1), 3-11. doi: 10.1016/0005-7916(87)90065-6.

Meier, T.B., Brummel, B.J., Singh, R., Nerio, C.J., Polanski, D.W., \& Bellgowan, P.S.F. (2015). The underreporting of self-reported symptoms following sports-related concussion. Journal of Science and Medicine in Sport, 18(5), 507-511. doi: 10.1016/j. jsams.2014.07.008.

Merritt, V.C., Meyer, J.E., \& Arnett, P.A. (2015). A novel approach to classifying postconcussion symptoms: The application of a new framework to the Post-Concussion Symptom Scale. Journal of Clinical and Experimental Neuropsychology, 37(7), 764-775. doi: 10.1080/13803395.2015.1060950.

Moser, R.S., Olek, L., \& Schatz, P. (2019). Gender differences in symptom reporting on baseline sport concussion testing across the youth age span. Archives of Clinical Neuropsychology, 34(1), 50-59. doi: 10.1093/arclin/acy007.

Nyer, M., Farabaugh, A., Fehling, K., Soskin, D., Holt, D., Papakostas, G.I., ... Mischoulon, D. (2013). Relationship between sleep disturbance and depression, anxiety, and functioning in college students. Depression and Anxiety, 30(9), 873-880. doi: $10.1002 /$ da. 22064 .

Peterson, R.A. \& Heilbronner, R.L. (1987). The anxiety sensitivity index. Journal of Anxiety Disorders, 1(2), 117-121. doi: 10.1016/ 0887-6185(87)90002-8.

Pratt, L.A., Druss, B.G., Manderscheid, R.W., \& Walker, E.R. (2016). Excess mortality due to depression and anxiety in the 
United States: Results from a nationally representative survey. General Hospital Psychiatry, 39, 39-45. doi: 10.1016/j. genhosppsych.2015.12.003.

Reiss, S., Peterson, R.A., Gursky, D.M., \& McNally, R.J. (1986). Anxiety sensitivity, anxiety frequency and the prediction of fearfulness. Behaviour Research and Therapy, 24(1), 1-8. doi: 10. 1016/0005-7967(86)90143-9.

Riegler, K.E., Guty, E.T., \& Arnett, P.A. (2019a). Validity of the ImPACT Post-Concussion Symptom Scale (PCSS) Affective symptom cluster as a screener for depression in collegiate athletes. Archives of Clinical Neuropsychology: The Official Journal of the National Academy of Neuropsychologists, 34(4), 563-574. doi: 10.1093/arclin/acy081.

Riegler, K.E., Guty, E.T., \& Arnett, P.A. (2019b). Neuropsychological test performance in depressed and nondepressed collegiate athletes following concussion. Neuropsychology. doi: 10.1037/neu00 00582 .

Riegler, K.E., Guty, E.T., \& Arnett, P.A. (2020). Neuropsychological test performance in depressed and nondepressed collegiate athletes following concussion. Neuropsychology, 34(1), 63-76. doi: 10.1037/neu0000582.

Riegler, K.E., Guty, E.T., Thomas, G.A., \& Arnett, P.A. (2021). Sleep deprived or concussed? The acute impact of self-reported insufficient sleep in college athletes. Journal of the International Neuropsychological Society: JINS, 27(1), 35-46. doi: 10.1017/S135561772000065X.

Sandin, B., Chorot, P., \& McNally, R.J. (2001). Anxiety sensitivity index: Normative data and its differentiation from trait anxiety. Behaviour Research and Therapy, 39(2), 213-219. doi: 10. 1016/S0005-7967(00)00009-7.

Simms, L.J., Prisciandaro, J.J., Krueger, R.F., \& Goldberg, D.P. (2012). The structure of depression, anxiety and somatic symptoms in primary care. Psychological Medicine, 42(1), 15-28. doi: 10.1017/S0033291711000985.

Snell, D.L., Martin, R., Macleod, A.D., Surgenor, L.J., Siegert, R.J., E. Jean, ... Anderson, T. (2018). Untangling chronic pain and post-concussion symptoms: The significance of depression. Brain Injury, 32(5), 583-592. doi: 10.1080/ 02699052.2018.1432894.

Solomon, G.S., Kuhn, A.W., \& Zuckerman, S.L. (2015). Depression as a modifying factor in sport-related concussion: A critical review of the literature. The Physician and Sportsmedicine. http://www.tandfonline.com/doi/abs/10.1080/ 00913847.2016.1121091

Suls, J. \& Howren, M.B. (2012). Understanding the physical-symptom experience: The distinctive contributions of anxiety and depression. Current Directions in Psychological Science, 21(2), 129-134. doi: 10.1177/0963721412439298.

Thomas, G.A., Guty, E.T., Riegler, K.E., \& Arnett, P.A. (2021). Comorbid affective symptomatology and neurocognitive performance in college athletes. Journal of the International Neuropsychological Society: JINS, 1-11. doi: 10.1017/ S1355617721000412.

Walczak, M., Ollendick, T., Ryan, S., \& Esbjørn, B.H. (2018). Does comorbidity predict poorer treatment outcome in pediatric anxiety disorders? An updated 10-year review. Clinical Psychology Review, 60, 45-61. doi: 10.1016/j.cpr.2017.12.005.

Wallace, J., Learman, K., Moran, R., Covassin, T., Deitrick, J.M., Delfin, D., \& Shina, J. (2020). Premorbid anxiety and depression and baseline neurocognitive, ocular-motor and vestibular performance: A retrospective cohort study. Journal of the Neurological Sciences, 418, 117110. doi: 10. 1016/j.jns.2020.117110.

Weber, M.L., Dean, J.-H.L., Hoffman, N.L., Broglio, S.P., McCrea, M., McAllister, T.W., . . Dykhuizen, B.H. (2018). Influences of mental illness, current psychological state, and concussion history on baseline concussion assessment performance. The American Journal of Sports Medicine, 46(7), 1742-1751. doi: 10.1177/ 0363546518765145.

Womble, M.N., Henley, J.R., Fedor, S.A., \& Collins, M.W. (2019). Posttraumatic headache and migraine assessment and management after sport-related concussion. In Neuropsychology of sports-related concussion (pp. 43-70). American Psychological Association. doi: 10.1037/0000114-003. 